\title{
Integrins Regulate Responsiveness to Slit Repellent Signals
}

\author{
Adrienne Stevens and J. Roger Jacobs \\ Department of Biology, McMaster University, Hamilton, Ontario, L8S 4K1, Canada
}

Integrins are concentrated within growth cones, but their contribution to axon extension and pathfinding is unclear. Genetic lesion of individual integrins does not stop growth cone extension or motility, but does increase axon defasciculation and axon tract displacement. In this study, we document a dosagedependent phenotypic interaction between genes for the integrins, their ligands, and the midline growth cone repellent, Slit, but not for the midline attractant, Netrin. Longitudinal tract axons in Drosophila embryos doubly heterozygous for slit and an integrin gene, encoding $\alpha \mathrm{PS} 1, \alpha \mathrm{PS} 2, \alpha \mathrm{PS} 3$, or $\beta \mathrm{PS} 1$, take ectopic trajectories across the midline of the CNS. Drosophila doubly heterozygous for slit and the genes encoding the integrin ligands Laminin A and Tiggrin reveal similar errors in midline axon guidance. We propose that the strength of adhesive signaling from integrins influences the threshold of response by growth cones to repellent axon guidance cues.

Key words: axon guidance; adhesion; extracellular matrix; Drosophila; midline; commissure
Integrin receptors provide a primary means of cell interaction with the extracellular matrix (ECM). Integrins bind adhesive molecules of the ECM, such as Laminin and Fibronectin, which communicate information for cell adhesion and movement (for review, see Cheresh and Mecham, 1994; Brown, 2000). Intracellularly, integrins provide the major linkage to the actin cytoskeleton, which mediates cell motility (for review, see Critchley et al., 1999). Integrin receptors are concentrated at the tips of filopodia in neuronal growth cones, where regulation of actin-based motility mediates axonal growth and pathfinding in development and regeneration (Grabham and Goldberg, 1997; Takagi et al., 1998).

Association of integrin receptors with ECM ligands is modeled to provide the physical linkage and adhesive signaling that stabilizes the actin cytoskeleton and enables growth cone extension. Integrin function is hypothesized to regulate the rate of axon growth (Letourneau, 1992; McKerracher et al., 1996; Condic and Letourneau, 1997). Axon growth may be facilitated by a low density of integrin adhesive contacts that can be formed and broken easily. In contrast, a high density or high binding affinity of integrin adhesive contacts could slow axon growth (Palecek et al., 1997; Maheshwari et al., 2000).

The large number of integrins in the mammalian CNS has hampered a genetic analysis of function in axonogenesis (Fassler et al., 1996; Hynes, 1996). Genetic data from mutations in the integrin genes of Caenorhabditis elegans and Drosophila do not suggest that integrins facilitate axon growth. Axon growth in these mutants is not noticeably slower, nor is pathfinding significantly altered (Baum and Garriga, 1997; Hoang and Chiba, 1998). Errors in passing innervation targets and axon defasciculation characterize the neuronal phenotype of these mutants. These phenotypes suggest a role for integrins in modulating adhesive signals, perhaps through regulated changes in adhesive

Received Dec. 19, 2001; revised Feb. 26, 2002; accepted March 5, 2002.

This work was supported by the Canadian Institutes for Health Research. We are grateful to Guy Tear, Tom Bunch, and Corey Goodman for providing essential reagents, and to Leena Patel for technical help.

Correspondence should be addressed to J. Roger Jacobs, Department of Biology, McMaster University, 1280 Main Street West, Hamilton, Ontario, L8S 4K1, Canada. E-mail: jacobsr@mcmaster.ca.

Copyright (C) 2002 Society for Neuroscience $\quad 0270-6474 / 02 / 224448-08 \$ 15.00 / 0$ affinity, and second messenger signaling to the cytoskeleton and other adhesion molecules (Hoang and Chiba, 1998).

In Drosophila integrin mutants, axon bypass errors occur at pathfinding checkpoints. Integrin adhesion or signaling may intersect with growth cone guidance signals at pathfinding choice points. These may be revealed by a genetic analysis of phenotypic interaction of mutations in the integrin genes and genes encoding growth cone attractants or repellents.

We have previously characterized the role of the growth cone repellent signal, Slit, in repressing the choice of commissural axon projection in axons of the longitudinal tracts (LTs) of the Drosophila embryo (Battye et al., 1999; Jacobs, 2000). Axons in embryos lacking slit function are attracted to the midline and do not leave. Increases or decreases in the level of slit expression in the midline of the Drosophila CNS correspondingly alter the number of axons that approach the midline (Battye et al., 2001). Midline repellent signaling is sensitive to gene dosage. For example, LT axons misproject across the midline in embryos with a single copy of the slit gene and a single copy of the gene encoding one of the receptors, Robo (Battye et al., 1999; Kidd et al., 1999).

If slit function is dose sensitive, a reduction in expression of other genes that affect midline axon guidance will reveal a phenotype in a heterozygous slit background. With this perspective, we have generated Drosophila doubly heterozygous for known mutations and slit and screened for errors in midline axon guidance. In this study, we provide genetic data to demonstrate that all integrins known to be expressed in the CNS are required to suppress errors in axon guidance. Furthermore, a penetrant dosage-dependent interaction of the genes for integrins and their ligands, with the midline repellent slit, suggests a role for integrins in regulating responsiveness to midline axon guidance signals.

\section{MATERIALS AND METHODS}

Drosophila strains. slit $^{2}$ (formerly slit $^{I G 107}$ ) was isolated by NüssleinVolhard et al. (1984), and slit ${ }^{2990}$ was isolated by Seeger et al. (1993). A wild-type genetic background was restored and then maintained as a $y w$, balanced strain. Loss of function alleles of $d o c k^{04723}$ and integrins $m y s^{1}$, $i^{k 27 c}, m^{2} w^{M 6}, s c b^{2}$, and hypomorphic $s c b^{01288}$ were obtained from the Indiana Stock Centre. The Netrin deficiency, $N P 5, r^{\circ} o^{1}$, and $r o b o^{3}$ were provided by G. Tear (King's College, London, UK) (Mitchell et al., 1996), lan $A^{9-32}$ was provided by C. Goodman (University of California, 
Berkeley, CA) (Garcia-Alonso et al., 1996), and $t^{x}{ }^{x}$ was provided by $\mathrm{T}$. Bunch (University of Arizona, Tucson, AZ) (Bunch et al., 1998).

Immunocytochemistry. Immunocytochemistry was adapted from Patel (1994). Embryos were collected at $22^{\circ} \mathrm{C}$ and fixed at $16 \mathrm{hr}$ intervals. Monoclonal antibody 1D4 [provided by N. Patel (University of Chicago, Chicago, IL) and C. Goodman] was diluted 1:4 in PBS with $0.1 \%$ Triton $\mathrm{X}-100$ and incubated at room temperature for $6 \mathrm{hr}$ followed by $2 \mathrm{hr}$ incubation in goat anti-mouse conjugated with HRP (Jackson ImmunoResearch, West Grove, PA) at a 1:1000 dilution. The presence of $P[$ lac Z $]$ balancer chromosomes was assessed with rabbit $\beta$-galactosidase antibody, 1:200 dilution (Cappel). Reactions with most embryos were performed in the presence of $0.03 \%$ cobalt chloride. Nerve cords were dissected in methyl salicylate before mounting in DPX (Sigma 31761-6) and visualized on a Zeiss Axiophot microscope. Phenotypes scored as percentages were determined from 90-200 segments scored in 12-25 embryos for each phenotype. The variance in the number of affected segments per embryo was comparable when the number of midline guidance errors was high (e.g., $s i^{2} / s c b^{2}$ was $5 \pm 2.1$ ) or low (e.g., mew/+; $s l i^{2} /+$ was $\left.0.7 \pm 1.6\right)$.

\section{RESULTS}

slit function is sensitive to dosage of interacting genes

Previous studies have demonstrated that mutations in slit interact phenotypically with mutations in robo, a gene encoding one of the Slit receptors (Battye et al., 1999; Kidd et al., 1999). The interaction is manifested as a reduction in midline repellent signaling, resulting in the misprojection of LT axons across the midline. These contralateral projections can be detected by assessing the distribution of Fasciclin II (Fas II)-labeled axons in the CNS at late embryogenesis. In wild-type embryos, Fas II is restricted to a bilateral trio of axon fascicles that maintain a constant distance from the midline (Fig. 1A). In the absence of midline repellent signals, as in slit loss-of-function mutants, all Fas II axons fuse into a single tract at the midline (Fig. 1B).

Three characteristic features of reduced midline signaling are evident in loss-of-function mutants for robo. Axons of the most medial Fas II fascicle cross and recross the midline, whereas lateral tracts are less affected (Fig. 1C, arrowhead). The intensity of Fas II labeling drops in LT axons between segments (Fig. 1C, arrow). Finally, the nervous system is narrower. Dock, a Drosophila ortholog of Nck, is an $\mathrm{SH} 2 / \mathrm{SH} 3$ adapter protein implicated in axon guidance signaling (Garrity et al., 1996). Embryos lacking dock function have a subtle Fas II phenotype, characterized by wavy LT fascicles, and interruptions in Fas II labeling in the lateral axon tracts (Fig. 1D). Although Drosophila with a single working copy of the slit, robo, or dock genes (heterozygous for a loss-of-function mutant) appear normal, novel axon guidance phenotypes emerge in embryos that have a single copy of two or more of these genes. Midline guidance errors are seen in $44 \%$ of segments in embryos with a single copy of the slit and robo genes (Fig. 1E) (all counts are summarized in Table 1). Embryos with a single copy each of the slit and dock genes have midline guidance errors in $77 \%$ of segments, sometimes involving all axon fascicles (Fig. $1 F$ ).

Changes in phenotype consistent with a change in gene copy number suggest gene function in a dosage-dependent event, such as signaling positional information in a gradient. Because Robo is a Slit receptor and Dock is a second messenger in the same or a parallel pathway, we hypothesize that the intensity of signaling by these molecules communicates positional information. Therefore, other genes that are required directly or indirectly to deliver this signal should also have a dosage-dependent phenotypic interaction with slit. We were interested in identifying other proteins on the cell surface or ECM that may interact with Slit and play a role in midline guidance. We therefore examined a number of muta- tions in genes encoding ECM proteins to determine whether they had a dosage-dependent phenotypic interaction with slit (Stevens, 2000). Mutations affecting integrin function revealed a dosagedependent interaction with slit, which is reported further here.

\section{Integrin mutations affect axon tracts}

We examined axon fasciculation and guidance in the CNS of embryos mutant for the $\beta$ integrin gene myospheroid (mys) and three $\alpha$ integrins, $\alpha \mathrm{PS} 1$ (mew), $\alpha \mathrm{PS} 2$ (if), and $\alpha \mathrm{PS} 3 / 4$ (scb). [It is not known whether the $s c b$ locus affects $\alpha \mathrm{PS} 3$ or $\alpha \mathrm{PS} 4$ or both, because the $\alpha$ PS4 locus is separated from $\alpha$ PS3 by 259 bp; Brown et al. (2000)]. Variation in penetrance of mutant phenotype was observed with all integrin alleles; representative examples of the more severe phenotypes are pictured here (Fig. $2 A-D$ ). The three longitudinal fascicles are intact in loss-of-function mutations in mys, which encodes the only $\beta$ integrin expressed in the CNS (Fig. $2 A)$; however, midline fusions of the most medial tract are seen in some segments. Axon tract structure was least disrupted in mew mutant embryos. No midline guidance errors were seen; however, the longitudinal fascicles appeared to be thinner, with occasional defasciculation (Fig. 2B, arrow). if mutant embryos were similar in phenotype to $m e w$, but also revealed a low frequency of midline crossover errors (Fig. 2C, arrowhead). Midline fusions as well as transient merging of lateral fascicles were seen in $s c b$ mutant embryos (Fig. 2D, arrow).

\section{Integrin mutants interact with slit}

Axon tract fasciculation appears normal in embryos heterozygous for mutations in one integrin gene and also in embryos heterozygous for mutations in two integrin genes (data not shown). However, all four integrin mutations reveal a semidominant phenotype when doubly heterozygous with slit. In all instances, the frequency of midline guidance errors is increased over the levels seen in homozygous integrin mutants. Apart from midline axon crossings in one-third of the segments, the LT appeared normal in mys $/+; s l i /+$ embryos (Fig. 2E, arrowhead; Table 1). Less than $10 \%$ of segments revealed midline guidance errors in $\mathrm{mew} /+$; sli/+ embryos (Fig. $2 F$ ). In contrast, 40 and $58 \%$ of segments had midline guidance errors in if $/+; s l i /+$ and $s c b / s l i$ embryos (Fig. $2 G, H$, respectively). Only in $s c b / s l i$ double heterozygotes were the middle and most lateral axon tracts affected, indicating a strong phenotypic interaction between scab and slit.

Midline guidance phenotypes were observed in integrin homozygotes that were also haplosufficient for slit. In particular, the frequency of midline crossing is much higher in mew/Y;sli/+ and if $/ \mathrm{Y} ; s l i /+$ mutants and also involves more lateral axon tracts (Fig. $2 J, K$, respectively).

We were not able to generate an $s c b$,sli recombinant to assess the $s c b, s l i / s c b$ phenotype. These genes would be expected to recombine in 1 of 25 chromosomes; however, we isolated no recombinants in 200 chromosomes screened. It was possible to investigate the interaction of these genes by using overlapping deficiencies. Midline guidance was assessed in $s c b$ and sli heterozygotes, in trans to a deficiency that uncovers either $s c b$ or $s l i$ or both genes (Fig. 3, schema). Consistent with our characterization of the $s l i$ and $s c b$ phenotypes, sli in trans to a deficiency uncovering sli has complete midline fusion of all axon tracts (Fig. $3 E, F)$, and $s c b$ in trans to a deficiency uncovering only $s c b$ has an integrin mutant phenotype (Fig. $3 A$ ). The semidominant interaction of $s l i$ and $s c b$ is also confirmed when $s c b$ is trans to a deficiency uncovering only slit (Fig. $3 B$ ) or when sli is trans to a deficiency uncovering only $s c b$ (Fig. $3 D$ ). A synthetic $s c b$ homozy- 


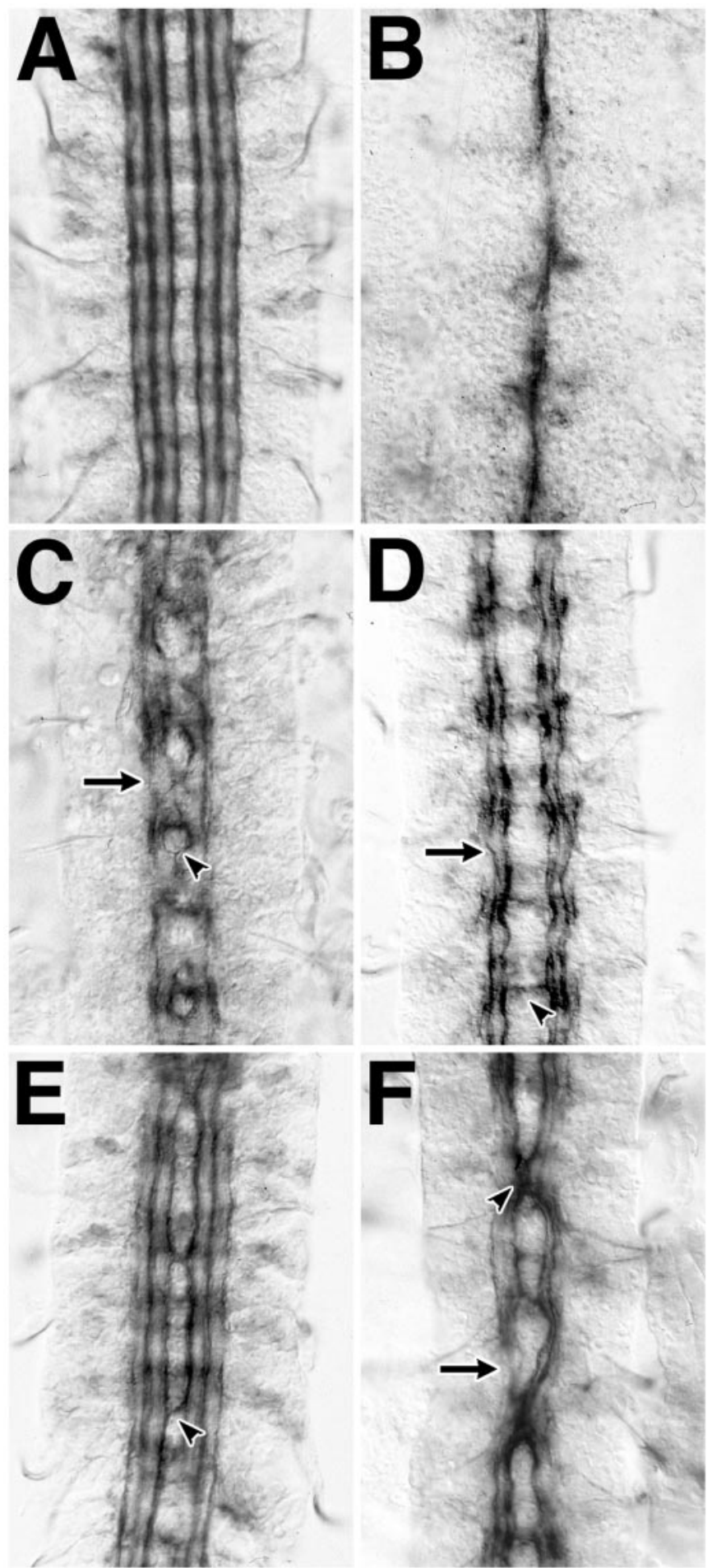

Figure 1. slit interacts genetically with robo and dock. A bilateral set of three distinct axon fascicles are labeled with antibody to Fasciclin II in stage 17 embryos of wild-type Drosophila $(A)$. All axons fuse at the midline in embryos mutant for slit $(B)$, whereas medial axons recross the midline in embryos mutant for a Slit receptor gene, robo ( $C$, arrowhead). Lateral axons do not make midline guidance errors, but occasional gaps in Fasciclin II labeling are seen $(C$, arrow). Midline guidance errors are very rare in embryos mutant for $\operatorname{dock}(D$, arrowhead $)$; however, gaps in Fasciclin II labeling in the lateral axon fascicles are common ( $D$, arrow). Midline guidance errors in the most medial axon tract are common in embryos that have a single wild-type and a single mutant copy of both the slit and robo genes ( $E$, arrowhead). Embryos similarly heterozygous for both slit and dock have more frequent and profound midline guidance gote and sli heterozygote phenotype was generated in embryos with a $s c b$ mutant allele in trans to a deficiency uncovering both $s c b$ and $s l i$. This phenotype is qualitatively similar to the $s c b / s l i$ phenotype, revealing frequent midline crossing or fusion of the two most medial but not the most lateral axon fascicles (Fig. 3C).

\section{Genes for integrin ligands interact with slit}

The midline axon phenotype of integrin mutants is part of a more complex phenotype involving defasciculation, irregular fascicle position, and "wavy" axon trajectories. This phenotype emerges even when slit function is normal and may reflect axon guidance functions of known integrin ligands in the nervous system. Two integrin ligands have been identified within the LT, Laminin, and Tiggrin (Montell and Goodman, 1989; Fogerty et al., 1994). We have characterized the Fas II phenotypes of embryos mutant for these ECM proteins to clarify their possible contribution to axon guidance.

Tiggrin is a secreted glycoprotein that contains an RGD motif and is considered to be a ligand of the PS2 integrin (Fogerty et al., 1994; Bunch et al., 1998). Embryos homozygous for a loss of function allele of Tiggrin have a subtle Fas II phenotype reminiscent of integrin mutants. CNS axon tracts are wavy, and no midline axon guidance errors are seen. Labeling of the most lateral axon tract is interrupted between segments (Fig. $4 A$, arrow). Like the integrin genes, tig also has a semidominant interaction with slit. Fas II labeling of fascicles between segments is reduced (Fig. 4B, arrow). Midline guidance errors are seen in one in three segments (Fig. 4B, arrowhead).

Drosophila Laminin is a trimer of three proteins, Laminin A, B1, and B2 (Montell and Goodman, 1989). Laminin is known to be a ligand of PS1 integrin and possibly other integrins as well (Gotwals et al., 1994). Mutants have not been isolated for the B1 and B2 chains; however, a loss of function allele for lan $A$ encoding the A chain has been characterized (Garcia-Alonso et al., 1996). The Fas II phenotype of the lanA mutant is nearly wild type, revealing midline guidance errors in $4 \%$ of segments (Fig. $4 C)(n=145$ segments). When doubly heterozygous with $s l i$, in sli/+;lanA/+ embryos, the frequency of midline crossovers is $>30 \%$ (Fig. 4D).

Does a change in lan $A$ function also affect integrin function in CNS axon tract formation? We chose to examine lanA interaction with $s c b$ because Laminin is not known to be a ligand of $\alpha \mathrm{PS} 3 / 4$ (encoded by $s c b$ ), and $s c b$ has a strong semidominant interaction with slit. Both lanA and $s c b$ reveal midline guidance errors when homozygous (Figs. $2 D, 4 C$ ). However, in the $s c b /+; l a n A /+$ double heterozygote, midline guidance errors are not seen (Fig. $4 E$ ). Nevertheless, this genotype shares aspects of the integrin CNS phenotype: defasciculation and interruptions in Fas II labeling of the most lateral fascicle (Fig. 4E, arrow). This suggests function of both genes in a common or parallel pathway. If the interaction of $s c b$ and $l a n A$ is independent of the interaction of either gene with $s l i$, then the phenotype of the triple heterozygote $s c b / s l i$; lan $A /+$ would reflect the addition of the $s c b / s l i, s c b /+; l a n A /+$, and sli/+;lan $A /+$ phenotypes. The degree of defasciculation and midline guidance errors in all axon tracts of the triple heterozygote (Fig. $4 F$ ) appears to be additive. However, a narrowing of the CNS and the medial displacement of all axon tracts are also seen

errors, involving all labeled axon fascicles $(F)$. In this and subsequent figures, arrowheads indicate midline guidance errors, and arrows indicate interruptions in the longitudinal tracts. 


\begin{tabular}{|c|c|c|c|c|c|c|c|c|c|c|c|c|c|}
\hline Genotype & $s l i /+$ & sli/robo & sli/dock & $\begin{array}{l}\text { mys/+; } \\
\text { sli/+ }\end{array}$ & $\begin{array}{l}\text { mew/+; } \\
\text { sli/+ }\end{array}$ & $\begin{array}{l}i f /+; \\
\text { sli/t+ }\end{array}$ & $s l i / s c b$ & $\begin{array}{l}\text { sli/+; } \\
\text { tig/+ }\end{array}$ & $\begin{array}{l}\text { sli/+; } \\
\text { lanA/++ }\end{array}$ & NP5/+ & $\begin{array}{l}\mathrm{NP5/+} \\
\mathrm{sli} /+\end{array}$ & $\begin{array}{l}\text { NP5/+; } \\
\text { robo/+ }\end{array}$ & $\begin{array}{l}\text { NP5/+; } \\
\text { scb/+ }\end{array}$ \\
\hline$\%$ Midline errors & 3.4 & 44 & 77 & 31 & 8.3 & 40 & 58 & 38 & 31 & 2.2 & 28 & 26 & 3.2 \\
\hline Number of segments & 146 & 127 & 110 & 90 & 96 & 104 & 108 & 92 & 99 & 138 & 198 & 120 & 92 \\
\hline
\end{tabular}

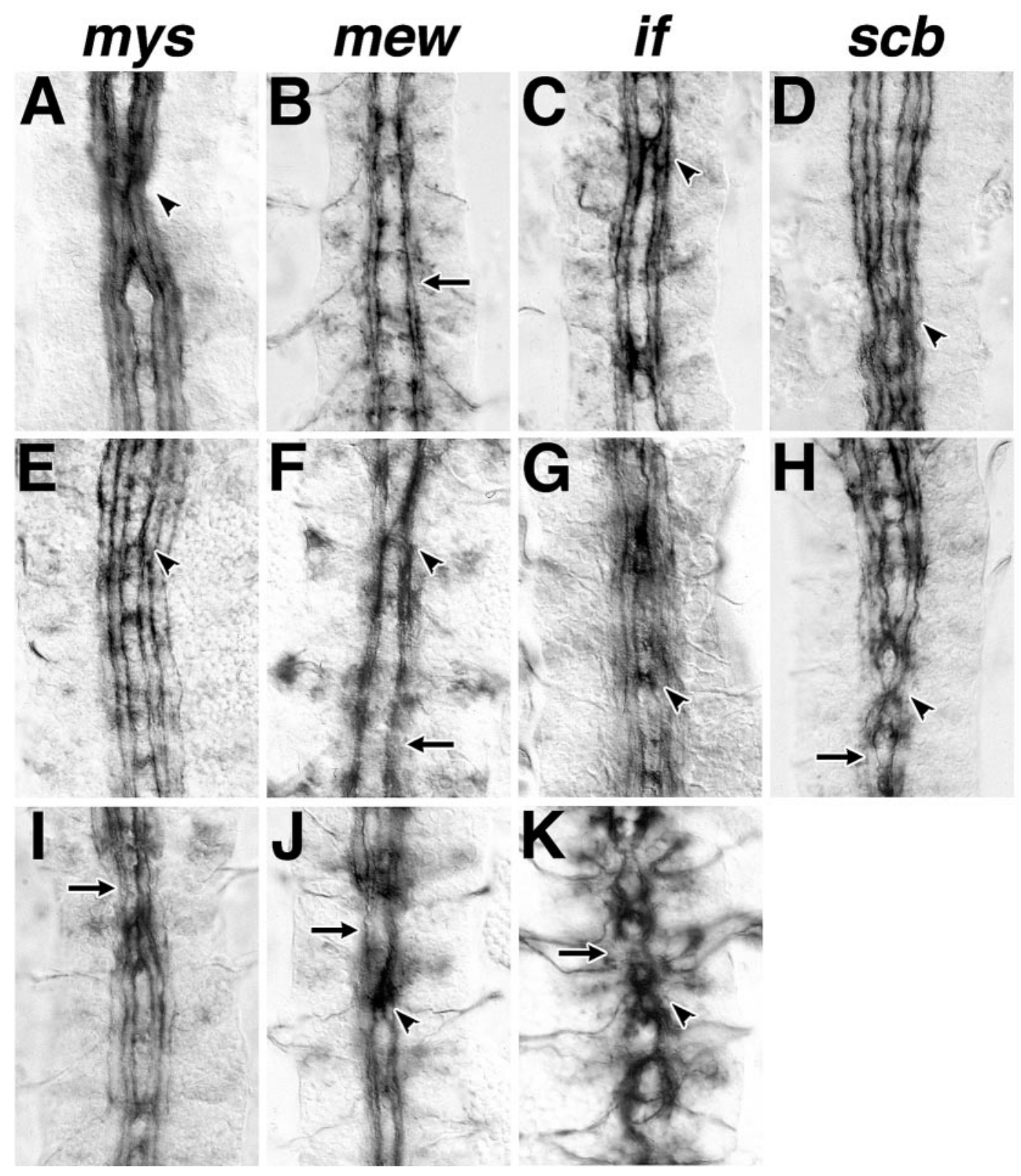

Figure 2. $\quad$ slit interacts genetically with integrin mutations. Embryos mutant for $\beta$-integrin $(A$, mys), $\alpha \mathrm{PS} 1$ integrin $(B$, mew $), \alpha \mathrm{PS} 2$ integrin $(C$, if $)$, and $\alpha$ PS3 integrin $(D, s c a b)$ have a range of axon guidance phenotypes; more severe examples are represented here. Midline errors (arrowheads) can be found in all integrin mutants; however, they occur more frequently and involve more axons in $s c a b$ and progressively fewer in if, mys, and mew mutant embryos. Axon guidance errors are seen in embryos heterozygous for both an integrin gene and slit (E, mys; $F$, mew; $G$, if; and $H$, scb) and in embryos homozygous mutant for an integrin gene and also heterozygous for slit ( $I$, mys; $J$, mew; and $K$, if). Midline axon crossover is most frequent in scb/sli heterozygotes and then less often in if, mys, and mew double heterozygotes, respectively.

in the triple heterozygote. This phenotype is typical of mutants in genes required for midline guidance and is not a component of the integrin mutant phenotype. The synergistic interaction of these three genes suggests dosage-dependent function for each gene in common or parallel pathways.

\section{Netrin interacts weakly with midline repellent genes}

Given that genes which function in cell to ECM adhesion interact with axon repellent signals, we wondered whether adhesion gene phenotypes interact similarly with midline attractant signals. We therefore explored whether embryos homozygous or heterozy- 


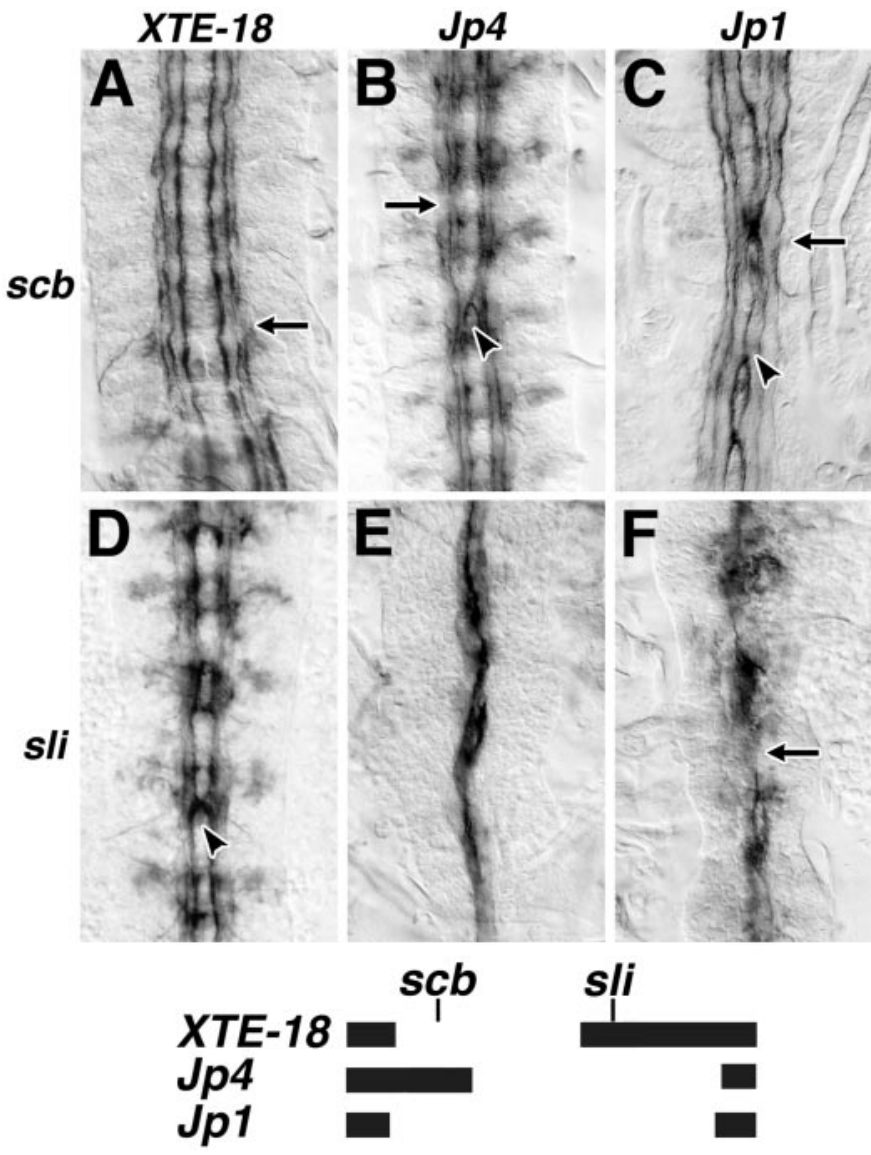

Figure 3. Phenotypic interaction of scab and sli. Midline guidance errors were assessed in embryos trans-heterozygous for either scab or sli and an overlapping deficiency. The extent of each deficiency is shown graphically below. Embryos heterozygous for $s c a b$ and a deficiency that does not uncover slit have a scab-like phenotype $(A, s c b / D f(2 R) X T E-18)$, and embryos heterozygous for slit and a deficiency that does uncover slit have a slit-like phenotype $(E, s l i / D f(2 R) J p 4 ; F$, sli/Df(2R)Jp1). The semidominant genetic interaction between sli and $s c a b$ is regenerated in $s c b /$ $D f(2 R) J p 4$ and sli/Df(2R)XTE-18 embryos ( $B$ and $D$, respectively). The medial Fascicilin II fascicles in $s c b / D f(2 R) J p 1$ embryos, which lack all scab function and are heterozygous for $s l i$, make midline guidance errors in all segments $(C$, arrowhead). More lateral fasicles are affected also $(C$, arrow).

gous for a deficiency that uncovers both Netrin genes, net $A$ and $n e t B$, affected midline guidance in a gene interaction assay. Embryos that lack netrin function have few commissural axons, and most commissures are missing (Mitchell et al., 1996). Embryos with one copy of each netrin gene $(\mathrm{NP5} /+)$ have normal commissures; however, the LTs, visualized with BP102, are thinner between segments and thicker within segments. Embryos that are heterozygous for mutations in both an integrin gene and the netrins show no enhancement or suppression of this phenotype (data not shown).

These data suggest that there may be a dosage-sensitive function of netrins in the organization of the LT. We chose to examine the morphology of Fasciclin II axon bundles more closely. Embryos homozygous or heterozygous for the netrin deficiency reveal irregularity and interruptions in longitudinal Fasciclin II bundles (Fig. 5A, $B$, respectively). The heterozygote netrin phenotype was not enhanced in embryos also heterozygous for slit, robo, or $s c a b$ function (Fig. $5 D-F$, respectively). In contrast, the frequency of midline guidance errors was increased in $s l i t /+$ or $r o b o /+$ but not $s c b /+$ embryos when netrin function was

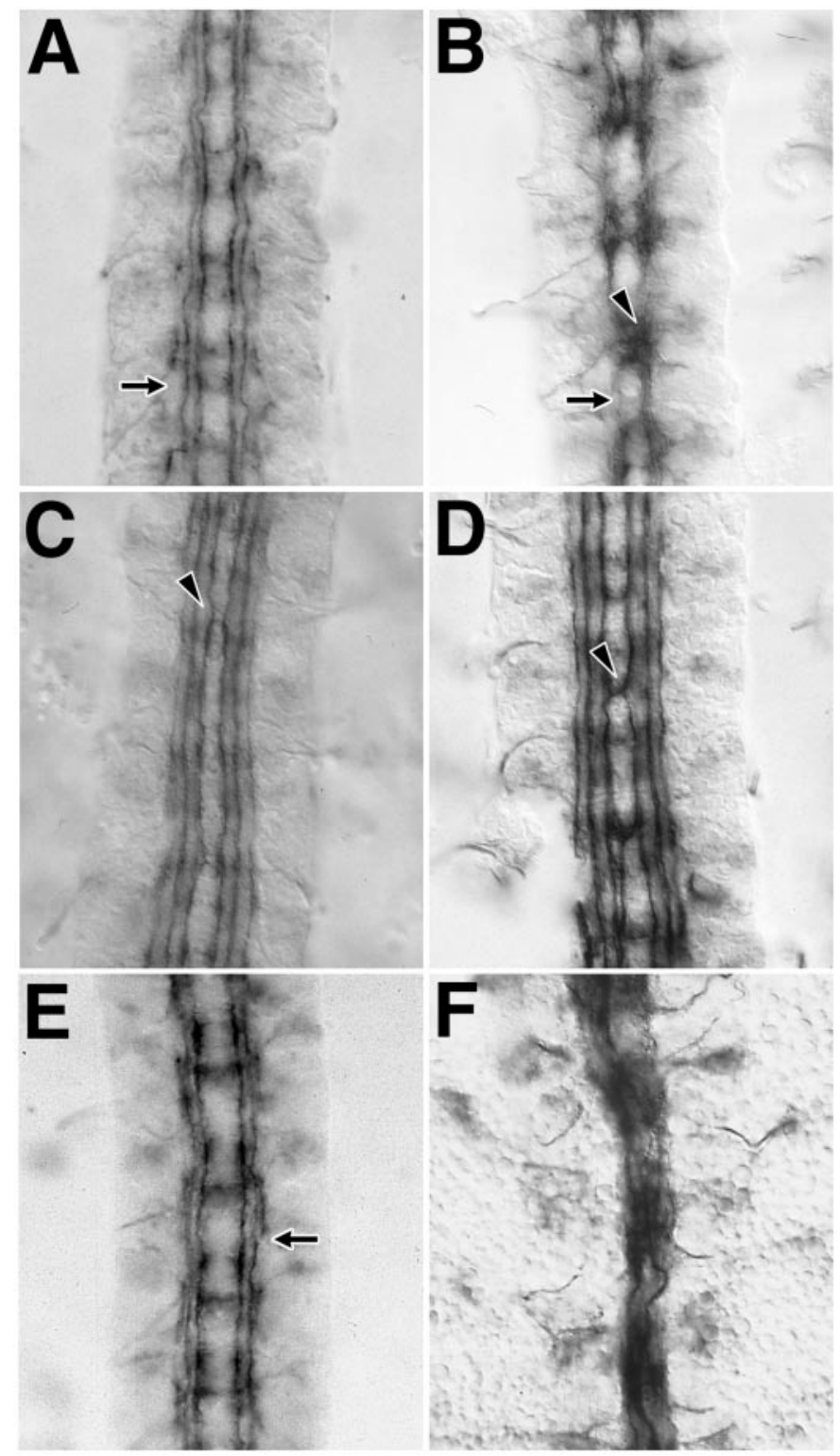

Figure 4. Integrin ligands interact genetically with $s c a b$ and slit. Embryos homozygous for tig have normal midline axon guidance but have wavy and undulating longitudinal tract fasicles $(A$, arrow). In embryos that are heterozygous for both tig and slit, fasciculation within the longitudinal tracts is abnormal ( $B$, arrow), and many Fas II-labeled axons project toward the midline (B,arrowhead). Embryos homozygous mutant for lan $A$ have a nearly normal Fas II labeling pattern, with rare midline guidance errors $(C$, arrowhead). Embryos heterozygous for both lan $A$ and slit are similar, but the frequency of midline guidance errors is higher $(D$, arrowhead). In embryos doubly heterozygous for both lan $A$ and $s c b$, defasciculations and gaps occur in the longitudinal tracts $(E$, arrow $)$. An embryo with a single copy of $s l i, s c b$, and $\operatorname{lan} A$ has pronounced medial displacement of all longitudinal tracts and midline fusion of medial axon tracts $(F)$.

also reduced or removed (Fig. 5D-F, arrowheads; Table 1). These data indicate that repellent signaling is significantly more dosage sensitive than attraction in midline phenotypes.

\section{DISCUSSION}

In this study, we have shown that a reduced level of expression of the genes for four integrins ( $\alpha \mathrm{PS} 1, \alpha \mathrm{PS} 2, \alpha \mathrm{PS} 3 / 4$, and $\beta \mathrm{PS} 1$ ) or two integrin ligands (Tiggrin and Laminin) increases the probability that CNS axons make pathfinding errors when slit expres- 


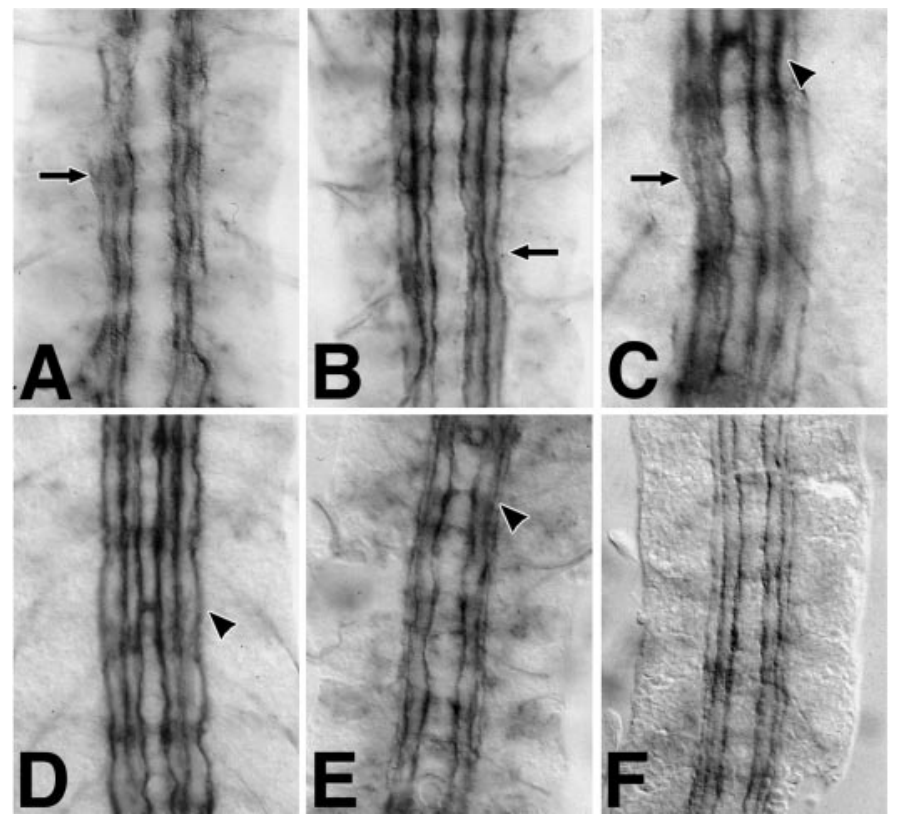

Figure 5. The netrin phenotype interacts weakly with slit. Fasciclin II labeling reveals defasciculation and gaps (arrow) in embryos homozygous for a small deficiency uncovering both net $A$ and netB $(A, N P 5 / Y)$. Embryos heterozygous for the Netrin deficiency have similar but less severe irregularities in Fasciclin II distribution $(B, N P 5 /+)$. Embryos lacking netrin function and heterozygous for slit reveal a netrin phenotype, with an increase in the frequency of midline guidance errors $(C, N P 5 / Y$; sli/+). Similarly, embryos doubly heterozygous for netrin and for slit $(D, N P 5 /+$; $s l i /+)$ or robo $(E, N P 5 /+$; robo/+) share the NP5/+ fasciclin II phenotype and a minor increase in the frequency of midline guidance errors $(D, E$, arrowheads). Midline guidance errors are not seen in netrin, scab double heterozygotes $(F, N P 5 /+; s c b /+)$.

sion is reduced. The netrin mutant phenotype was not affected by reduced integrin function.

\section{Axon tract phenotypes of integrins and their ligands}

Expression of the integrins Tiggrin and Laminin A has been demonstrated previously in the CNS (Montell and Goodman, 1989; Fogerty et al., 1994; Hoang and Chiba, 1998; Rohrbough et al., 2000). Integrin expression is not localized and may be expressed in both glia and neurons. Overexpression of $\alpha \mathrm{PS} 3$ or Laminin A in motoneurons affects axon guidance (Kraut et al., 2001). Loss of function of the integrins disrupts axon fasciculation and longitudinal axon fascicle placement in the embryonic nerve cord but does not clearly affect axon guidance (Hoang and Chiba, 1998). In this study, we extended these observations with different alleles of the integrins, demonstrated a similar function for $\alpha \mathrm{PS} 3 / 4$, and revealed axon fascicle phenotypes for loss of function of integrin ligands Tiggrin and Laminin A. The mutant phenotypes share common elements: mild phenotypes show wavy axon tracts and reduced Fas II labeling between segments, whereas severe phenotypes include defasciculation and fascicle displacement, including midline axon guidance errors. The integrins have different extracellular ligands. Therefore, the integrins contribute similarly to axon tract integrity, independent of the ligand that they bind.

Integrin phenotypes in the CNS do not demonstrate a direct role for integrins in growth cone guidance. In contrast, perturbation of midline growth cone repellent signals results in a medial narrowing of the CNS and ectopic midline crossing of longitudinally projecting axons, rather than defasciculation and displace- ment of axon tracts. One feature of integrin and tiggrin phenotypes shared with robo and dock mutant phenotypes is a thinning or loss of Fas II labeling in the most lateral axon fascicle. This fascicle expresses Fas II late in embryogenesis. This phenotype may reflect impaired or delayed development of independent fascicles in the nerve cord, as implicated by other studies of robo function (Rajagopalan et al., 2000; Simpson et al., 2000).

\section{The nature and significance of genetic interaction with axon guidance signals}

Axon guidance cues such as Netrin or Slit are secreted proteins that associate with the ECM. Vertebrate Slit, for instance, binds to Laminin, Netrin, and Glypican (Brose et al., 1999; Liang et al., 1999). These cues also act at a distance from the cells that synthesize them. Whether or not Slit forms a detectable gradient in the ECM, the amount of protein alters the potency of repellent signaling. A robo-like phenotype is seen in hypomorphs of slit that produce less protein, and overproduction of slit reduces the number of commissural axons (Battye et al., 1999, 2001); therefore, Slit signaling is dosage sensitive. If reduced expression of another gene enhances the midline guidance phenotype of slit, then the normal function of that gene contributes functionally to inhibit axons from crossing the midline. This may reflect function in the production or transduction of the repellent signal or another function in the growth cone that reduces the probability of a growth cone approaching the midline.

The semidominant interaction of all integrins, Tiggrin, and Laminin A with slit is more prevalent than might be expected if the integrins play a specialized role in Slit signaling. scab also has a dramatic semidominant interaction with dock (Nck), which functions in diverse axon guidance events (Garrity et al., 1996; Hing et al., 1999). scb/dock double heterozygotes have disrupted longitudinal, commissural, and peripheral axon tracts (J. R. Jacobs, unpublished observations). A similar genetic test suggests that $\beta$ PS integrin modulates RhoA activity and axon stability in the mushroom body (Billuart et al., 2001). These diverse phenotypes reflect an adhesive function of the integrins that reduces the responsiveness of growth cones to guidance signals. Independent evidence suggests that this occurs in the growth cone, but a role for integrin in the glia that emit guidance signals cannot be discounted.

Mutations in the netrin $A$ and netrinB genes $\operatorname{did}$ not reveal semidominant interactions with genes for integrin function. Therefore Netrin signaling is not dosage sensitive in this genetic assay. Although Netrin might form a gradient in vivo, these data suggest that Slit may more effectively communicate positional information than does Netrin. Double mutants of netrin and slit have a slit phenotype, indicating that Netrin signaling acts genetically upstream of repulsion and also that attraction to the midline persists in the absence of Netrin (Jacobs, unpublished observations). These data suggest that Netrin is not the sole midline attractant in Drosophila. More axons approach the midline in a netrin, slit double heterozygote than would if only slit function is reduced. Therefore Slit and Netrin do not generate independent, additive guidance signals. Netrin can bind to Slit (Brose et al., 1999). Furthermore, the Slit receptor may silence attractant signaling by the Netrin receptor (Stein and Tessier-Lavigne, 2001). Copresentation of Slit and Netrin to the receptors on the growth cone may enhance the repellent signal. Attraction to the midline requires silencing of Slit signaling. In Drosophila, repellent signals can be silenced by Comm, which facilitates internalization of the Robo receptor (Kidd et al., 1998). 


\section{How do integrins contribute to axon guidance?}

Integrins are concentrated in the growth cones of Drosophila axons (Takagi et al., 1998; Takagi et al., 2000), and their ligands are uniformly distributed over pathways of axon extension, (Montell and Goodman, 1989; Fogerty et al., 1994). Integrins facilitate the growth of axons by providing a link between the ECM and the cytoskeleton of the growth cone. Defasciculation and guidance errors seen in integrin mutants reflect decreased adhesion to the ECM and a lower threshold to errors in guidance. Axon extension is not impaired in Drosophila integrin mutants, although it is possible that a maternal contribution of integrin may mask this requirement.

Part of the adhesive function of integrins is to activate intracellular signals that alter motility and axon outgrowth. During ligand binding, $\beta$ integrins may activate Focal Adhesion Kinase and Rho, which stabilize actin structures, permit actin filament growth, and facilitate the formation of focal adhesions (Clark et al., 1998; Bishop and Hall, 2000). Intracellular signals can also modify adhesiveness by altering the affinity of integrins for their ligands. These signals can combine to cluster integrins and strengthen their attachment to the ECM. This increased adhesiveness can act in opposition to factors that decrease adhesion and axon extension, such as myelin or aggrecan (David et al., 1995). Furthermore, integrin signaling can influence other adhesion systems active in the growth cone (Hemler, 1998; Arregui et al., 2000; Iba et al., 2000).

Similarly, integrin function alters the sensitivity of growth cones to repellent signaling by Slit. When slit expression and integrin function are both reduced, growth cones are more likely to respond to attractive guidance from the midline. Signals from axon guidance receptors promote growth cone reorientation and remodeling of the growth cone cytoskeleton. Integrin adhesion promotes local stabilization of cytoskeletal links to the ECM, which antagonizes growth cone reorientation. The threshold of response of growth cones to axon guidance signals is therefore regulated by the ability of guidance signals to reduce the stability of ECM to cytoskeletal linkages. This threshold may be reached at axon guidance choice points, including the segment boundary and the commissures, where clustering of errors in axon guidance occur. The efficacy of guidance signals may be reduced between choice points by local increases in ECM affinity.

Integrin-ligand affinity and integrin-cytoskeletal linkages are logical targets of axon guidance signals. Further studies of the targets of integrin and guidance signals should reveal how growth cones integrate information from the ECM.

\section{REFERENCES}

Arregui C, Pathre P, Lilien J, Balsamo J (2000) The nonreceptor tyrosine kinase fer mediates cross-talk between N-cadherin and beta1integrins. J Cell Biol 149:1263-1274.

Battye R, Stevens A, Jacobs JR (1999) Axon repulsion from the midline of the Drosophila CNS requires slit function. Development 126:24752481.

Battye R, Stevens A, Perry RL, Jacobs JR (2001) Repellent signaling by Slit requires the leucine-rich repeats. J Neurosci 21:4290-4298.

Baum PD, Garriga G (1997) Neuronal migrations and axon fasciculation are disrupted in ina-1 integrin mutants. Neuron 19:51-62.

Billuart P, Winter CG, Maresh A, Zhao X, Luo L (2001) Regulating axon branch stability. The role of p190 RhoGAP in repressing a retraction signaling pathway. Cell 107:195-207.

Bishop AL, Hall A (2000) Rho GTPases and their effector proteins. Biochem J 348:241-255.

Brose K, Bland KS, Wang K-H, Arnott D, Henzel W, Goodman CS, Tessier-Lavigne M, Kidd T (1999) Evolutionary conservation of the repulsive guidance function of Slit proteins and their interactions with robo receptors. Cell 96:795-806.
Brown NH (2000) Cell-cell adhesion via the ECM: integrin genetics in fly and worm. Matrix Biol 19:191-201.

Brown NH, Gregory SL, Martin-Bermudo MD (2000) Integrins as mediators of morphogenesis in Drosophila. Dev Biol 223:1-16.

Bunch TA, Graner MW, Fessler LI, Fessler JH, Schneider KD, Kerschen A, Choy LP, Burgess BW, Brower DL (1998) The PS2 integrin ligand tiggrin is required for proper muscle function in Drosophila. Development 125:1679-1689.

Cheresh D, Mecham R (1994) Integrins: molecular and biological responses to the extracellular matrix. San Diego: Academic.

Clark EA, King WG, Brugge JS, Symons M, Hynes RO (1998) Integrinmediated signals regulated by members of the rho family of GTPases. J Cell Biol 142:573-586.

Condic ML, Letourneau PC (1997) Ligand-induced changes in integrin expression regulate neuronal adhesion and neurite outgrowth. Nature 389:852-856

Critchley DR, Holt MR, Barry ST, Priddle H, Hemmings L, Norman J (1999) Integrin-mediated cell adhesion: the cytoskeletal connection. Biochem Soc Symp 65:79-99.

David S, Braun PE, Jackson DL, Kottis V, McKerracher L (1995) Laminin overrides the inhibitory effects of peripheral nervous system and central nervous system myelin-derived inhibitors of neurite growth. J Neurosci Res 42:594-602.

Fassler R, Georges-Labouesse E, Hirsch E (1996) Genetic analyses of integrin function in mice. Curr Opin Cell Biol 8:641-646.

Fogerty FJ, Fessler LI, Bunch TA, Yaron Y, Parker CG, Nelson RE, Brower DL, Gullberg D, Fessler JH (1994) Tiggrin, a novel Drosophila extracellular matrix protein that functions as a ligand for Drosophila alpha PS2 beta PS integrins. Development 120:1747-1758.

Garcia-Alonso L, Fetter RD, Goodman CS (1996) Genetic analysis of laminin A in Drosophila: extracellular matrix containing laminin A is required for ocellar axon pathfinding. Development 122:2611-2621.

Garrity PA, Rao Y, Salecker I, McGlade J, Pawson T, Zipursky SL (1996) Drosophila photoreceptor axon guidance and targeting requires the dreadlocks SH2/SH3 adapter protein. Cell 85:639-650.

Gotwals PJ, Fessler LI, Wehrli M, Hynes RO (1994) Drosophila PS1 integrin is a laminin receptor and differs in ligand specificity from PS2. Proc Natl Acad Sci USA 91:11447-11451.

Grabham PW, Goldberg DJ (1997) Nerve growth factor stimulates the accumulation of $\beta 1$ integrin at the tips of filopodia in the growth cones of sympathetic neurons. J Neurosci 17:5455-5465.

Hemler ME (1998) Integrin associated proteins. Curr Opin Cell Biol 10:578-585

Hing H, Xiao J, Harden N, Lim L, Zipursky SL (1999) Pak functions downstream of Dock to regulate photoreceptor axon guidance in Drosophila. Cell 97:853-863.

Hoang B, Chiba A (1998) Genetic analysis on the role of integrin during axon guidance in Drosophila. J Neurosci 18:7847-7855.

Hynes RO (1996) Targeted mutations in cell adhesion genes: what have we learned from them? Dev Biol 180:402-412.

Iba K, Albrechtsen R, Gilpin B, Frohlich C, Loechel F, Zolkiewska A, Ishiguro K, Kojima T, Liu W, Langford JK, Sanderson RD, Brakebusch C, Fassler R, Wewer UM (2000) The cysteine-rich domain of human ADAM 12 supports cell adhesion through syndecans and triggers signaling events that lead to beta1 integrin-dependent cell spreading. J Cell Biol 149:1143-1156.

Jacobs JR (2000) The midline glia of Drosophila: a molecular genetic model for the developmental functions of Glia. Prog Neurobiol 62:475-508.

Kidd T, Russell C, Goodman CS, Tear G (1998) Dosage-sensitive and complementary functions of roundabout and commissureless control axon crossing of the CNS midline. Neuron 20:25-33.

Kidd T, Bland K, Goodman CS (1999) Slit is the midline repellent for the Robo receptor in Drosophila. Cell 96:785-794.

Kraut R, Menon K, Zinn K (2001) A gain-of-function screen for genes controlling motor axon guidance and synaptogenesis in Drosophila. Curr Biol 11:417-430.

Letourneau P (1992) Integrins and N-cadherin are adhesive molecules involved in growth cone migration. In: The nerve growth cone (Letourneau P, Kater S, Macagno E, eds), pp 181-193. New York: Raven.

Liang Y, Annan RS, Carr SA, Popp S, Mevissen M, Margolis RK, Margolis RU (1999) Mammalian homologues of the Drosophila slit protein are ligands of the heparan sulfate proteoglycan glypican-1 in brain. J Biol Chem 274:17885-17892.

Maheshwari G, Brown G, Lauffenburger DA, Wells A, Griffith LG (2000) Cell adhesion and motility depend on nanoscale RGD clustering. J Cell Sci 113:1677-1686.

McKerracher L, Chamoux M, Arregui CO (1996) Role of laminin and integrin interactions in growth cone guidance. Mol Neurobiol 12:95-116.

Mitchell KJ, Doyle JL, Serafini T, Kennedy TE, Tessier-Lavigne M, Goodman CS, Dickson BJ (1996) Genetic analysis of Netrin genes in Drosophila: Netrins guide CNS commissural axons and peripheral motor axons. Neuron 17:203-215.

Montell D, Goodman C (1989) Drosophila Laminin: sequence of B2 
subunit and expression of all three subunits during embryogenesis. J Cell Biol 109:2441-2453.

Nüsslein-Volhard C, Wieschaus E, Kluding H (1984) Mutations affecting the pattern of the larval cuticle in Drosophila melanogaster I. Zygotic loci on the second chromosome. Roux's Arch Dev Biol 193:267-282.

Palecek SP, Loftus JC, Ginsberg MH, Lauffenburger DA, Horwitz AF (1997) Integrin-ligand binding properties govern cell migration speed through cell-substratum adhesiveness. Nature 385:537-540.

Patel N (1994) Imaging neuronal subsets and other cell types in wholemount Drosophila embryos and larvae using antibody probes. Methods Cell Biol 44:445-505.

Rajagopalan S, Vivancos V, Nicolas E, Dickson BJ (2000) Selecting a longitudinal pathway: Robo receptors specify the lateral position of axons in the Drosophila CNS. Cell 103:1033-1045.

Rohrbough J, Grotewiel MS, Davis RL, Broadie K (2000) Integrinmediated regulation of synaptic morphology, transmission, and plasticity. J Neurosci 20:6868-6878.
Seeger M, Tear G, Ferres-Marco D, Goodman CS (1993) Mutations affecting growth cone guidance in Drosophila: genes necessary for guidance toward or away from the midline. Neuron 10:409-426.

Simpson JH, Bland KS, Fetter RD, Goodman CS (2000) Short-range and long-range guidance by Slit and its Robo receptors: a combinatorial code of Robo receptors controls lateral position. Cell 103:1019-1032.

Stein E, Tessier-Lavigne M (2001) Hierarchical organization of guidance receptors: silencing of netrin attraction by slit through a Robo/ DCC receptor complex. Science 291:1928-1938.

Stevens AL (2000) Dosage dependent modifiers of slit mutants. Masters thesis, McMaster University.

Takagi Y, Ui-Tei K, Miyake T, Hirohashi S (1998) Laminin-dependent integrin clustering with tyrosine-phosphorylated molecules in a Drosophila neuronal cell line. Neurosci Lett 244:149-152.

Takagi Y, Ui-Tei K, Hirohashi S (2000) Adhesion-dependent tyrosine phosphorylation of enabled in Drosophila neuronal cell line. Biochem Biophys Res Commun 270:482-487. 